\title{
ON THE $p$-MIXED AFFINE SURFACE AREA
}

\author{
XIAO-YAN LI AND CHANG-JIAN ZHAO
}

Abstract. Some new inequalities for $i$-th mixed $p$-affine surface area are established. The results in special cases yield some of the recent results on inequalities of this type.

Mathematics subject classification (2010): 52A20.

Keywords and phrases: Affine surface area, mixed $p$-affine surface area, Brunn-Minkowski inequality.

\section{REFERENCES}

[1] T. Bonnesen, W. Fenchel, Theorie der konvexen Körper, Springer-Verlag, Berlin, 1934.

[2] E. LutwaK, Mixed affine surface area, J. Math. Anal. Appl., 125 (1987), 351-360.

[3] R. J. Gardner, Geometric Tomography, Cambridge Univ. Press, Cambridge, 1995.

[4] R. SchneIder, Convex Bodies: The Brunn-Minkowski Theory, Cambridge Univ. Press, 1993.

[5] E. LutwaK, The Brunn-Minkowski-Firey theory II: Affine and geominimal surface areas, Adv. Math., 118 (1996), 244-294.

[6] W. Wang, G. Leng, $L_{p}$-mixed affine surface area, J. Math. Anal. Appl., 335 (2007), 341-354.

[7] E. LutwaK, The Brunn-Minkowski-Firey theory I: Mixed volumes and the Minkowski problem, J. Diff. Geom., 38 (1993), 131-150.

[8] E. LutwaK, Extended affine surface area, Adv. Math., 85 (1991), 39-68.

[9] E. F. Bechenbach, R. Bellman, Inequalities, Springer-Verlag, Berlin-Göttingen, Heidelberg, 1961.

[10] G. H. Hardy, J. E. Littlewood and G. Pólya, Inequalities, Cambridge Univ. Press. Cambridge, 1934.

[11] M. Ludwig, M. Reitzner, A characterization of Affine surface area, Adv. Math., 147 (1999), 138172.

[12] E. WeRnER, Illumination bodies and affine surface area, Studia Math., 110 (1994), 257-269.

[13] D. Hug, Contributions to affine surface area, Manuscripta Mathematica, 91 (1996), 283-301.

[14] C. ZhaO, G. LENG, Width-integrals of projection bodies and affine surface area, Chinese Ann. Math., 26 (2005), 275-282.

[15] Y. D. Burago, V. A. Zalgaller, Geometric Inequalities, Springer-Verlag, Berlin, 1988.

[16] M. Meyer, E. Werner, On the p-affine surface area, Adv. Math., 152 (2) (2000), 288-313.

[17] C. SChÜTt, E. WeRnER, Surface bodies and p-affine surface area, Adv. Math., 187 (2004), 98-145.

[18] E. Werner, D. Ye, New $L_{p}$ affine isoperimetric inequalities, Adv. Math., 218 (2008), 762-780.

[19] E. Werner, D. YE, Inequalities for p-affine surface area, Math. Ann., 347 (2010), 703-737. 